\title{
Prognosis of patients with apparent treatment-resistant hypertension-a feasibility study
}

Peter Hayes ${ }^{1 *}$, Hannah Kielty ${ }^{2}$, Monica Casey ${ }^{1}$, Liam G. Glynn ${ }^{3}$, Gerard J. Molloy ${ }^{4}$, Hannah Durand ${ }^{4}$, John Newell ${ }^{5}$ and Andrew W. Murphy ${ }^{1,6}$

\begin{abstract}
Background: Most cases of hypertension can be effectively treated with lifestyle changes together with medications, but within this population lies a group with more difficult to treat hypertension - those with apparent treatment-resistant hypertension (aTRH). The American Heart Association and the UK National Institute for Health and Care Excellence have both highlighted the need for further research into the prognosis of patients with resistant hypertension, both apparent and true.
\end{abstract}

Methods: In 16 practices affiliated to a university research network, 646 patients had been identified with apparent treatment-resistant hypertension. To inform a planned full cohort study of these patients, we conducted a feasibility study within three practices to determine participation of practices and patients, availability of outcome measures and data collection times.

Results: All three practices fully participated and 205/210 (98\%) patients were followed up for a median of 23 months. Thirty-five outcome events of interest occurred - the most common was the new onset of retinopathy (9 cases). Eight percent (17/210) had the main composite outcome of death or serious incident cardiovascular event. Of the six patients who died, identification of cause of death was possible from practice records in five; the national General Register Office was successfully used for the final patient. There were 123 admissions, both day and overnight, recorded in 94 individual patients. Average manual systolic blood pressure measurements improved from baseline by $5 \mathrm{mmHg}$ to 138 (SD 19) $\mathrm{mmHg}$; diastolic remained the same at 75 (SD 12) $\mathrm{mmHg}$. Average eGFR increased from $58.8(\mathrm{SD} 17.4)$ to $66(\mathrm{SD} 19.7) \mathrm{mls} / \mathrm{min} / 1.73 \mathrm{~m}^{2}$. The average time for data collection per patient was 12 mins.

Conclusions: This study demonstrates that the proposed methodology for a full cohort study within general practice of patients with apparent treatment hypertension is both acceptable to practices and feasible. An adequately powered subsequent follow-up study of the entire cohort appears possible.

Keywords: Hypertension, Primary care, Treatment resistant hypertension, Prognosis, Feasibility

\footnotetext{
* Correspondence: peter.hayes@nuigalway.ie

${ }^{1}$ Discipline of General Practice, School of Medicine, National University of

Ireland, Galway, Ireland

Full list of author information is available at the end of the article
} 


\section{Background}

Arterial hypertension accounts for, or contributes to, $62 \%$ of all strokes and $49 \%$ of all cases of heart disease [1]. Most cases of hypertension can be effectively treated with lifestyle changes and/or medications, but within this population lies a group with more difficult to treat hypertension-those with treatment-resistant hypertension (TRH). TRH is defined as high blood pressure in a patient taking three or more differing groups of antihypertensive medications (one of which must be a diuretic type medication) or any patient who is taking four or more anti-hypertensive medications irrespective of blood pressure level [2]. Doses should be the optimal tolerated doses for each particular medication, lifestyle issues should also be addressed and white coat hypertension needs to be examined by ambulatory blood pressure monitoring (ABPM). When issues of dosing, medication adherence and white coat hypertension have not yet been ruled out, the term apparent treatment-resistant hypertension (aTRH) is utilised.

A recent meta-analysis of the prevalence of aTRH for a total population of 961,035 patients with diagnosed hypertension yielded prevalences for observational studies and trials respectively of 13.7 and $16.3 \%$ [3]. Individual study prevalence varied between 4.2 and $25.4 \%$. Having established that resistant hypertension is apparently common, a key next step is to determine how important it is. Multiple cross-sectional studies comparing patients with aTRH to those without have suggested increased frequency of target organ damage and later cardiovascular complications [4]. In a key study, Daugherty [5] provided the best evidence to date with an outcomebased study on the longitudinal assessment of a large cohort of patients with aTRH $(n=3960)$. She found a $50 \%$ increase in cardiovascular events (largely attributable to development of chronic kidney disease) compared to those whose blood pressure was controlled on three medications. This impressive study is however a retrospective study of electronic insurance data with no assessment of ABPM or dosing and limited assessment of adherence. Sim also confirmed a possible increased cardiovascular risk associated with aTRH in 60,327 patients [6]. Patients with resistant hypertension, compared to those without, had significantly increased hazard ratios for stroke and mortality, respectively, of 1.14 (95\% CI 1.10-1.19) and 1.06 (95\% CI 1.03-1.08). However, Irvin [7] in retrospectively comparing similar groups found no significant increased risk of stroke but an increased risk for mortality. These studies, utilising large electronic databases, had large patient numbers but limited assessment of adherence, dosing and white coat hypertension. The American Heart Association [8] and the UK National Institute for Health and Care Excellence [9] have both highlighted the need for further high-quality prospective research into the prognosis of patients with resistant hypertension.

Previously, in a cross-sectional study of 6691 patients with hypertension in 16 Irish general practices, we identified 646 patients in Irish general practice with aTRH, whose files were individually reviewed and in whom pseudo-resistance was also examined [10]. These 646 patients therefore represent a unique general practice cohort of patients with comprehensive assessment for true treatment-resistant hypertension. It is planned to conduct a future prognostic cohort study of these 6691patients to compare those with apparent treatmentresistant hypertension and those with essential hypertension. In anticipation of this, we conducted a feasibility study to determine participation of practices and patients, availability and frequency of outcome measures and data collection times required.

\section{Methods \\ Design and setting}

The original cross-sectional study has been comprehensively described elsewhere [10]. In short,16 general practices in the university-affiliated research network WestREN, representative of the Irish population, participated [11]. All used the same practice software system (Socrates $^{\odot}$ ) and the International Classification for Primary Care (ICPC-2) coding of chronic diseases. Ireland does not have universal registration with a general practitioner. All patients aged over 70 years, or below 6 years, and those below defined income levels receive free general practitioner care. These patients represent almost $40 \%$ of the general population and are registered with the Primary Care Reimbursement Service (PCRS; http:// www.hse.ie/eng/staff/PCRS/) with the remainder being described as private patients and able to see any general practitioner. We therefore included in the crosssectional sample all PCRS patients and those private patients who had attended the practice in the last year.

Each practice ran a standard ATC drug search identifying patients on any possible hypertensive medications as defined by the British National Formulary 69th Edition (https://www.amazon.co.uk/British-NationalFormulary-BNF-69/dp/0857111566). Two researchers (PH and $\mathrm{MC}$ ), in conjunction with the practices, then reviewed the record of each individual patient who was reported as being on one or more hypertensive medications and determined if they were hypertensive or not, had a previous ambulatory blood pressure measurement or not and what hypertensive medications and doses they were currently receiving. This work fulfilled for the general practitioners, the Irish Medical Council requirement, to conduct an annual audit.

Patients were then identified as being hypertensive if this diagnosis was recorded in clinical notes by their GP 
or if they had the appropriate diagnosis code recorded in the patient file (i.e. International Classification of Primary Care codes for hypertension-K87, K87). No start date for the diagnosis was recorded; this is not therefore an incident cohort.

\section{Recruitment}

For this feasibility study, three practices were pragmatically chosen from the original 16 to reflect practice diversity-one was small and rural, one a medium and rural practice and one a large and mixed urban-rural practice. All 16 practices used the same practice software system $\left(\right.$ Socrates $\left.^{\circ}\right)$ to store patient data, were located within 1-h travel of the university and had a mix of PCRS and private patients.

There were no incentives provided to these practices to participate; however, a stipend of $€ 1000$ was paid to all 16 practices when assembling the initial cohort as an acknowledgement for the extra workload involved. All 210 patients in these three practices were eligible to participate.

\section{Data collection}

In each of the three practices, a manual review of each patient's individual patient electronic record was performed by two trained researchers (HK and MC).

Both researchers participated in seminars on resistant hypertension. Training was also provided on how to use the practice software system (Socrates ${ }^{\circ}$ ) and more specifically on how to search for the data required. Both researchers worked in one practice together, and then, HK completed the other two practices, recording any issues for later clarification.

\section{Outcomes}

We recorded the time spent in each practice for data collection, the proportions of patients whose records could be successfully accessed and who had an outcome of interest.

The proposed main composite outcome measurement is the same as that used by Daugherty [5]: all-cause mortality and incident cardiovascular events which include nonfatal myocardial infarction, heart failure, stroke or chronic kidney disease (CKD). Identification of cause of death was primarily through hospital or physician notices in patient's files or through death certs. Where these were not informative, the national online General Register Office was utilised https://www.welfare.ie/en/ Pages/Apply-for-Certificates.aspx.

Identification of the specified conditions are based on diagnosis codes, hospital correspondence and lab data. Patients are described as having diabetes if they have ICPC codes T89 (diabetes insulin-dependent) or T90 (diabetes non-insulin-dependent) or are taking insulin or oral hypoglycaemic agents. Patients are described as having CKD if an estimated glomerular filtration rate (eGFR) less than $60 \mathrm{mls} / \mathrm{min} / 1.73 \mathrm{~m}^{2}$ was recorded. Patients are described as having cardiac failure if they have ICPC code K77 or are noted to have this condition on hospital correspondence.

Recent blood pressure (BP) readings (manual and 24-h ambulatory) were also recorded, as was antihypertensive medication and doses. Hospital admissions and diagnoses were identified by discharge summaries. Day admissions largely involved medical or surgical assessment unit visits, angiograms or specialised testing requiring a ward admission. We did not include patients presenting for simpler tests such as echocardiograms or stress tests as a day admission.

\section{Power calculation}

Table 3 illustrates the outcome events for patients with apparent treatment-resistant hypertension over a median of 1.9 years. Daugherty et al., for their main composite outcome over 3.8 years, found that 18 and $13.5 \%$ of patients with apparent treatment hypertension and simple hypertension respectively had the outcome. $8.3 \%$ of our cohort had the equivalent of Daugherty's composite outcome and $17 \%$ a combination of all cardiovascular events. This suggests that a review of our entire original cohort over 4 years will result, at least, in similar outcome proportions to those of Daugherty.

Taking our original cohort of 6045 patients with simple hypertension and 646 patients with aTRH, allowing for a loss to follow-up of $10 \%$ and applying the proportions of 18 and $13.5 \%$, with a significance level of 0.05 , confirms that the cohort has $80 \%$ power to show a significant difference.

\section{Statistics analysis plan}

The primary response is the probability of patients developing the outcome of interest as defined by Daugherty. A logistic regression model will be used to compare the probabilities between the two groups while adjusting for explanatory variables such as age, gender, socioeconomic status, baseline blood pressure, presence of diabetes or kidney disease as appropriate. Initially all explanatory variables will be included as adjusters, and the ridge regression and the LASSO [12] will be used to account for any multicollinearity present among the explanatory variables. Classification trees will be used to identify potentially useful interactions.

\section{Results}

All three contacted practices agreed to participate. Of the original 210 patients in these practices, data was available for 205 patients (97.6\%). Five patients had transferred practice and data was not available (see Fig. 1). Follow-up was for a median of 23 months. File 


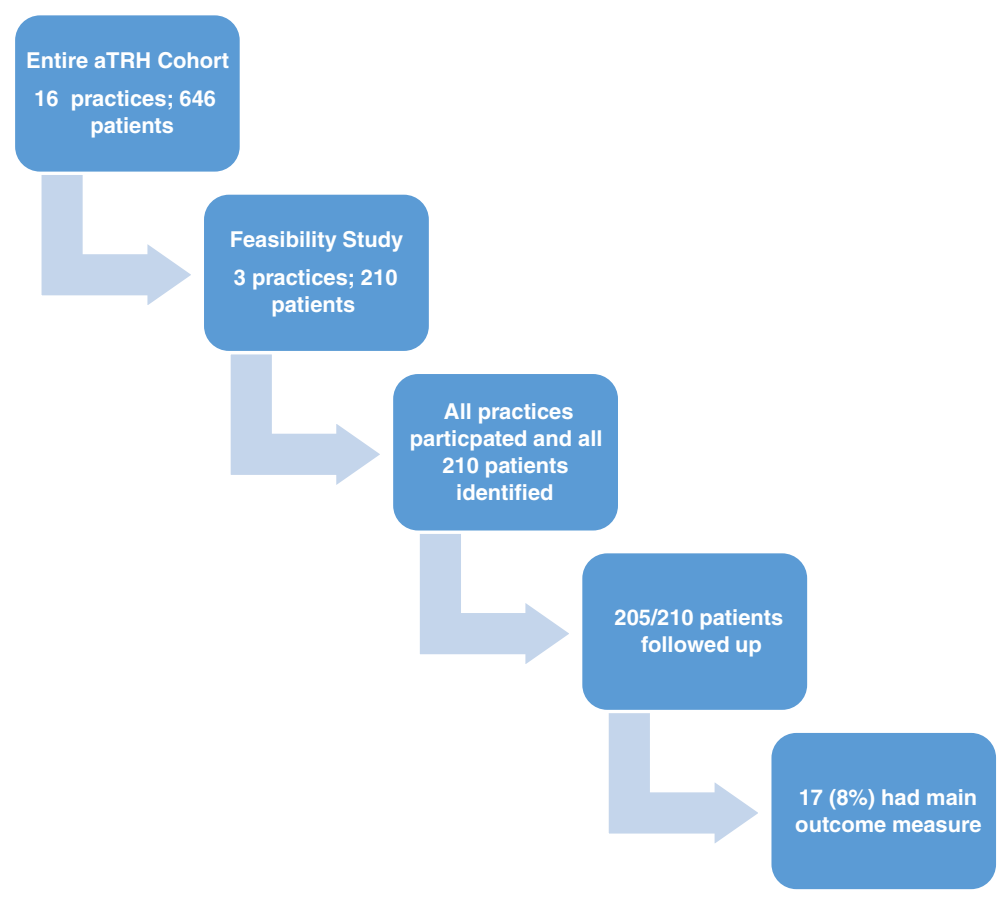

Fig. 1 Flow sheet of participants in the feasibility study

search took place from June 19 to July 142017 -a total of 4 weeks for three practices. The total time spent in data collection at the practices was $44 \mathrm{~h}$ which was an average of $12.6 \mathrm{~min}$ per patient.

Table 1 shows the characteristics of the original cohort $(n=646)$, and Table 2 illustrates the characteristics of the 205 patients examined for prognosis. The average manual BP reading was $138 \mathrm{mmHg}$ (SD19) systolic blood pressure and $75 \mathrm{mmHg}$ (SD12) diastolic blood pressure. A mean reduction of $5 \mathrm{~mm} \mathrm{Hg}$ systolic $(\mathrm{SD} 11 \mathrm{mmHg})$ is seen between the latest clinic readings and the original cohort's readings. One hundred twelve patients (over the previous 23 months median follow-up period) had ABPM, and the daytime average ABPM

Table 1 Demographic and clinical details for original cross sectional group $(n=646)$

\begin{tabular}{lll}
\hline Demographic & (Mean if applicable) & Standard deviation (from mean) \\
\hline Age (years) & 71.1 & 12 \\
Sex & $54 \%$ male & \\
Eligible for Primary Care Reimbursement Scheme (marker of household income) & $82 \%(n=530)$ & \\
Clinical details & $37 \%(n=237)$ & $18 / 12 \mathrm{mmHg}$ \\
Diabetes & $40 \%(n=258)$ & $21 / 14 \mathrm{mmHg}$ \\
CKD & $12 \%(n=79)$ & 0.7 \\
Cardiac failure & $142 / 78 \mathrm{mmHg}$ & $148 / 81 \mathrm{mmHg}(n=74)$ \\
$\quad$ Manual BP & & \\
Recent ABPM BP (where available within 6 months) & 3.7 \\
Medications & $56 \%(n=364)$ & \\
$\quad$ No. of anti-hypertensive drugs used & & \\
4 or more anti-hypertensive drugs & 166 \\
Sub-divisions & 480 \\
$\quad$ True TRH & & \\
Pseudo-resistant TRH & & \\
\hline
\end{tabular}


Table 2 Demographic and clinical details for patients with apparent treatment-resistant hypertension in feasibility study $(n=210)$

\begin{tabular}{lll}
\hline Demographic & (Mean if applicable) & Standard deviation (from mean) \\
\hline Age (years) & 73.7 YOA & 11.2 \\
Sex & $56 \%$ male & \\
Eligible for Primary Care Reimbursement Scheme (marker of household income) & $80 \%(n=167)$ & \\
Clinical details & $31 \%(n=65)$ & $19 / 12 \mathrm{mmHg}$ \\
Diabetes & $41 \%(n=87)$ & $16 / 10 \mathrm{mmHg}$ \\
CKD & $15 \%(n=31)$ & 1.06 \\
Cardiac failure & $138 / 75 \mathrm{mmHg}$ & $134 / 76 \mathrm{mmHg}(n=112)$ \\
$\quad$ Manual BP & & \\
Recent ABPM BP (where available within 23 months median follow-up period) & 3.7 \\
Medications & $51 \%$ & \\
No. of anti-hypertensive drugs used & & \\
4 or more anti-hypertensive drugs & $26 \%(\mathrm{n}=65)$ & $74 \%(n=140)$ \\
Subdivisions & & \\
True TRH & & \\
Pseudo-resistant THR & & \\
\hline
\end{tabular}

reading was 136 (SD16)/74 (SD10) $\mathrm{mmHg}$. There were anti-hypertensive medications and or dose changes in $57 \%$. Nine percent $(n=18)$ no longer met the minimum inclusion criteria for aTRH of three or more medications. Average eGFR increased from 58.8 (SD17.4) to 66 (SD19.7) $\mathrm{mls} / \mathrm{min} / 1.73 \mathrm{~m}^{2}$. From the 210 patients examined, 65 persons have true resistant TRH (26\%) and 140 persons have pseudo-resistant TRH (Table 2), while from the main cohort of 646 persons with aTRH, 30\% $(n=166)$ have true TRH and 70\% $(n=480)$ have pseudoresistant TRH (Table 1).

Thirty-five events of interest occurred-the most common was the new-onset retinopathy at 4.3\% (9/210) (see Table 3). Of the six patients who died, identification of

Table 3 Outcome events for patients with apparent treatmentresistant hypertension in feasibility study $(n=205)$

\begin{tabular}{ll}
\hline Any cause of death $^{a}$ & 6 \\
CVD cause death & 1 \\
New non-fatal myocardial infarction $^{\mathrm{a}}$ & 2 \\
New non-fatal congestive cardiac failure $^{\mathrm{a}}$ & 5 \\
New non-fatal cerebrovascular accident $^{\mathrm{a}}$ & 3 \\
New-onset chronic kidney disease $^{\mathrm{a}}$ & 1 \\
New non-fatal transient ischaemic attack & 5 \\
New-onset retinopathy & 9 \\
New atrial fibrillation & 3 \\
New onset peripheral arterial disease & 0 \\
Total & 35 \\
Number of events total & 17 \\
Number of individuals with composite outcome & \\
\hline
\end{tabular}

${ }^{\mathrm{a}}$ These are included in the main outcome composite outcome (Daugherty) cause of death was possible from practice records in five; the national General Register Office was successfully used for the final patient. Seventeen patients from 210 in the examined cohort $(8 \%)$ had the main composite outcome of death or an incident cardiovascular event of nonfatal myocardial infarction, heart failure, stroke or CKD. There were 123 admissions recorded in 94 individual patients, both day and overnight, and these are detailed in Table 4.

\section{Discussion}

Prior to conducting a larger cohort study of all 646 patients in all 16 practices, we needed to demonstrate the feasibility of the proposed methodology by addressing key uncertainties. Our key uncertainties related to the acceptability of follow-up to practices, the availability of outcome data (especially in a health system without universal registration) and the workload involved. By explicitly addressing these uncertainties in a feasibility study, we can confirm that an adequately powered subsequent follow-up study of the entire cohort is possible.

We attempted to ensure that the follow-up strategy imposed little burden on practices, and this appeared to work well. The average research time of $12 \mathrm{~min}$ per

Table 4 Admissions for patients with apparent treatmentresistant hypertension in feasibility study (123 events, 94 patients)

\begin{tabular}{lll}
\hline Admissions & Number $(n=123)$ & Percentage of total \\
\hline Non-CVD hospitalisations & 62 & $50 \%(62 / 123)$ \\
Non-CVD day admissions & 27 & $22 \%(27 / 123)$ \\
CVD hospitalisations & 23 & $19 \%(23 / 123)$ \\
CVD day admissions & 11 & $9 \%(11 / 123)$ \\
\hline
\end{tabular}


patient includes data collection only, but travel to practices was a mean of $80 \mathrm{~min}$ return (maximum trip- $2 \mathrm{~h}$ return). The main variation in data collection time between practices was the number of patient files examined. The main variation in data collection time between individual patients was the number of recorded health care interactions.

Tips for ensuring a smooth research process include pre-booking time slots for practice computers, showing flexibility regarding access times; multiple half days may be available as opposed to full days and administrator/ front desk workload acknowledgement, e.g. teas, courtesy and thank you cards. The signing of individual practice confidentiality agreements was also seen as important. These may not be required by local ethics committees, but GP's data protection fears are eased by such. Tips for ensuring accurate data collection include ensuring the adequate training of researchers in use of the relevant patient's data management system and meeting with practice administrators prior to data collection. Administrators will know where specific data items are stored, e.g. ABPM reports and how the files of those who are deceased or have moved on are managed in individual practices. These issues will need to be factored into future planning.

All practices that participated were part of a university-affiliated research network WestREN. Regular e-mail updates and annual meetings where research projects were discussed are key to maintaining practice participation. Allowing practices to fulfil their mandatory audit commitments, while participating in research projects, is also welcome. The very low numbers lost to follow-up and completeness of data are pleasing; however, this may not be replicated in the larger level, and due allowance has been made in the power calculation.

Outcome measurement data appeared readily available. Coding of individual consultations is not common in Irish general practice. A combination of coding for the key diagnoses of interest by practices, together with review of scanned written hospital discharges, while time-consuming, appeared to overcome this challenge. In the last 5 years, public hospitals in the region have introduced electronic discharge summaries which automatically enter the individual patient electronic record, significantly aiding the outcome assessment process. Identification of cause of death from practice data also appeared straightforward with only one of six deaths requiring access to the online State Register. This was needed for an in-hospital patient death, where cause of death was unclear from practice clinical notes and notice of death, as opposed to cause of death, was recorded.

Two outcome measurements were of particular interest. New-onset retinopathy has not been reported in other similar cohort studies. It was our most common outcome event. With over a third of our patients with apparent resistant hypertension having diabetes, this is of potential significance. Consideration may need to be given to including retinopathy in any composite outcome measurement. It highlights the importance of the work of the COMET (Core Outcomes Measures in Effectiveness Trials) group who are agreeing standardised sets of outcomes (http://www.comet-initiative.org). There is, as yet, no agreed core outcome set in the study of resistant hypertension. Contrary to our expectations, renal function as estimated by eGFR, improved rather than deteriorated over the duration of follow-up. Clearly, our sample size limits the interpretation of this. However, it does emphasise the need for serial eGFR measurements to be utilised rather than 'once off' recordings.

\section{Comparison with other work}

Table 5 illustrates the key parameters of previous studies reviewing the prognosis of patients with aTRH. Our study is distinguishable as both the only study based in general practice and the only study to have individual patient record review. This facilitates consideration of dosing, adherence and white coat hypertension which are often overlooked in other studies. Similar to Daugherty, we will be able to, with adequate power, compare outcomes for patients with simple hypertension to those with treatment-resistant hypertension (Table 2). However, the numbers with true resistant hypertension will be small making meaningful comparisons difficult.

\section{Strengths and limitations}

We based our methodology on a previous cohort study we conducted in the region which followed up 1605 patients with heart disease from 35 practices after 5 years [13]. This experience was invaluable in developing our protocol. The subsequent introduction of automatic hospital electronic discharges and enhanced coding by practices has aided cohort study conduct.

There are four significant limitations. Firstly, on balance, a non-random selection of practices was preferred as it allowed us to sample the diversity of practice size and location. We used three typical practices. It also facilitated transport issues for researchers, within a short available time period, to be taken into account. Other practices may have specific issues which we have not encountered, but the recording of electronic health care data is similar in the Socrates ${ }^{\oplus}$ practice software system, which is reassuring.

Secondly, the Irish health system is a complex amalgam of private and public providers. Other similar studies (Daugherty [5], Irvin [7]) had the significant benefits of an integrated health system facilitating collection of primary, secondary and tertiary care data. We can never be sure that some important outcome data is not 
Table 5 Comparison between prognostic studies of patients with apparent treatment-resistant hypertension

\begin{tabular}{lllll}
\hline Study & Setting & Record & Duration of follow-up & $\begin{array}{l}\text { Pseudo-resistance } \\
\text { considered }\end{array}$ \\
\hline Daugherty (2012) [5] & Primary/secondary care health insurance & Electronic database & Median 3.8 years & No \\
Sim et al. (2015) [6] & Primary/secondary care health insurance & Electronic database & Retrospective cohort 5 years & No \\
Irvin et al. (2014) [7] & Population-based cohort recruited & Patient interview & Median 4.4-6 years & No (adherence-yes) \\
Hayes et al. (planned 2020) & Primary care & Manual file search & Median 5 years & Yes \\
\hline
\end{tabular}

missing. Thirdly, we did not follow up the five patients who transferred practice. This may be the main challenge in the future and will likely involve locating patients who have moved practice. These patients may subsequently have their data now recorded in an alternative electronic format. Consideration in the full cohort study to providing resources to do this is appropriate. Finally, the cohort was assembled in a cross-sectional study and not as an incident cohort with no recorded date of diagnosis.

\section{Conclusions}

This study demonstrates that the proposed methodology for a full cohort study within general practice of patients with apparent treatment hypertension is both acceptable to practices and feasible. An adequately powered subsequent follow-up study of the entire cohort appears possible.

\section{Abbreviations \\ ABPM: Ambulatory blood pressure monitoring; ATC: Anatomical therapeutic chemical; aTRH: Apparent treatment-resistant hypertension; BP: Blood pressure; Cl: Confidence interval; CKD: Chronic kidney disease; eGFR: Estimated glomerular filtration rate; SD: Standard deviation; tTRH: True treatment-resistant hypertension}

\section{Acknowledgements}

The authors are indebted to all participating patients and general practices and staff of the Department of General Practice at the National University of Ireland, Galway, where the study was coordinated.

\section{Funding}

This feasibility study was funded by the Department of General Practice, National University of Ireland, Galway. The original study funding was awarded by the Health Research Board (Reference number HRA-POR-2014-615). The funder had no role in the design of the study design or the manuscript.

\section{Availability of data and materials}

Not applicable.

\section{Authors' contributions}

AWM is the principal investigator and, with $\mathrm{PH}$, led the pilot study design and funding application. PH is the team lead and contributed to the study design. MC is the project manager/researcher. HK is a medical student at NUI Galway and carried out the data collection. GM is a co-applicant and led on the adherence aspect of the original study with $\mathrm{HD}$ as a PhD candidate. JN, a co-applicant, is the study statistician and contributed to the statistical aspects of the protocol. LG is a co-applicant on the original HRB funded study. All authors contributed to, read and approved the final version of the manuscript.

\section{Ethics approval and consent to participate}

This study was approved by the Clinical Research Ethics Committee at Merlin Park University Hospital in November 2015 Reference number C.A. 1386.
Consent for publication

Not applicable.

\section{Competing interests}

Two of the authors (AWM, LG) have received funding in the past from MSD pharmaceutical company.

\section{Publisher's Note}

Springer Nature remains neutral with regard to jurisdictional claims in published maps and institutional affiliations.

\section{Author details}

'Discipline of General Practice, School of Medicine, National University of Ireland, Galway, Ireland. ²School of Medicine, NUI Galway, Galway, Ireland.

${ }^{3}$ Graduate Entry Medical School, University of Limerick, Limerick, Ireland.

${ }^{4}$ School of Psychology, NUI Galway, Galway, Ireland. ${ }^{5}$ School of Mathematics, Statistics and Applied Mathematics, NUI Galway, Galway, Ireland. ${ }^{6}$ Health Research Board Primary Care Clinical Trials Network, Galway, Ireland.

Received: 25 September 2017 Accepted: 10 January 2018

Published online: 30 January 2018

\section{References}

1. Myat A, et al. Resistant hypertension. BMJ. 2012;345:e7473

2. Calhoun DA, et al. Resistant hypertension: diagnosis, evaluation, and treatment. A scientific statement from the American Heart Association Professional Education Committee of the Council for High Blood Pressure Research. Hypertension. 2008;51(6):1403-19.

3. Achelrod D, Wenzel U, Frey S. Systematic review and meta-analysis of the prevalence of resistant hypertension in treated hypertensive populations. Am J Hypertens. 2015;28(3):355-61.

4. Pimenta E, Calhoun DA. Resistant hypertension: incidence, prevalence, and prognosis. Circulation. 2012;125(13):1594-6.

5. Daugherty $\mathrm{SL}$, et al. Incidence and prognosis of resistant hypertension in hypertensive patients. Circulation. 2012;125(13):1635-42.

6. Sim JJ, et al. Comparative risk of renal, cardiovascular, and mortality outcomes in controlled, uncontrolled resistant, and nonresistant hypertension. Kidney Int. 2015;88(3):622-32.

7. Irvin MR, et al. Apparent treatment-resistant hypertension and risk for stroke, coronary heart disease, and all-cause mortality. J Am Soc Hypertens. 2014; 8(6):405-13.

8. Calhoun DA, et al. Resistant hypertension: diagnosis, evaluation, and treatment: a scientific statement from the American Heart Association Professional Education Committee of the Council for High Blood Pressure Research. Circulation. 2008;117(25):e510-26.

9. (NICE), T.N.I.I.H.a.C.E., Hypertension: clinical management of primary hypertension in adults. NICE clinical guideline 127., 2011.

10. Hayes P, C. M, Glynn LG, Molloy GJ, Durand H, O' Brien E, Dolan E, Newell J, Murphy AW. The prevalence of treatment resistant hypertension in general practice with consideration of pseudo-resistance and morbidity. BJGP. 2018 (In Press)

11. Kavanagh $K E$, et al. WestREN: a description of an Irish academic general practice research network. BMC Fam Pract. 2010;11:74.

12. Hastie T, T. R., Friedman J., Springer series in statistics. 2008.

13. Glynn LG, et al. Multimorbidity and risk among patients with established cardiovascular disease: a cohort study. BJGP. 2008;58(552):488-94. 